\title{
ROBOTARIUMS IMPLEMENTATIONS WITH THE ELEMENTS OF ARTIFICIAL INTELLIGENCE CONTROL
}

\author{
Aryskin A.A., Davydov O.I., Eprikov S.R., Ksenzenko A.I., Pryanichnikov V.E., \\ Punenkov O.V., Shipovalov E.A, Solovyova M.D., Stepanova D.K., \\ Tarasov R.B. \& Tikhomirov A.N.
}
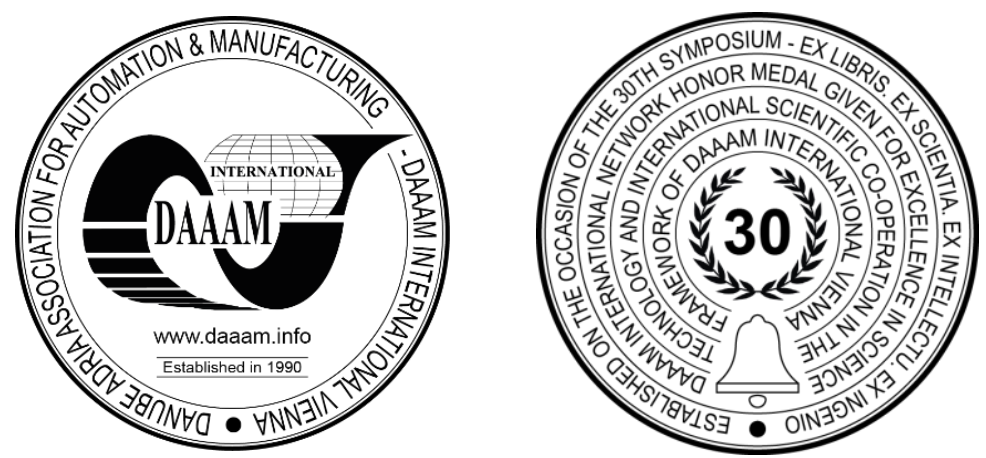

This Publication has to be referred as: Aryskin, A[lexander]; Davydov, O[leg]; Eprikov S[tanislav]; Ksenzenko A[lexander]; Pryanichnikov, V[alentin]; Punenkov O[leg]; Shipovalov E[gor]; Solovyova M[ariia]; Stepanova D[aria]; Tarasov R[adomir] \& Tikhomirov A[lexey] (2020). Robotariums implementations witn the Elements of Artificial Intelligence Control, Proceedings of the 31st DAAAM International Symposium, pp.0973-0985, B. Katalinic (Ed.), Published by DAAAM International, ISBN 978-3-902734-29-7, ISSN 1726-9679, Vienna, Austria DOI: $10.2507 / 31$ st.daaam.proceedings. 135

\begin{abstract}
The aim of the research was to increase the autonomy and flexibility of mobile robotic systems, develop effective algorithms of path finding of service robots, operating in rooms surrounded by people, and create technologies for intellectualization and unification, including their applications for industrial systems and underwater robotics. The paper describes a control system, that allows you to implement several modes of supervisory control in robotariums. Network integration software made it possible to unify the control of heterogeneous groups of robots both in the mode of their digital counterparts and directly with the real objects. The mobile robot AMUR-307 has been developed, which is planned to be used in clinics to move various objects between the premises of the building. A hardware and software system was designed and manufactured to control robots of this type, and its comprehensive testing was performed. The solution allows you quickly make changes, following the customer's requirements and deliver robots, integrating them into the existing infrastructure. The article also analyzes the possibility use new type of planetary-tipping gearbox for the manipulator of the AMUR-307 service robot and on a walking underwater vehicle. The trajectory of movement of the output link of an eccentric-cycloidal gearing with a gear ratio of 1:20 is studied. The trajectory of movement of the output link of the Chebyshev mechanism, while using an eccentric-cycloidal gearing, is analyzed.
\end{abstract}

Keywords: mobile service robots; intelligent robotronics; interpretation of lidar and odometer readings; pass-frames; eccentric-cycloidal gearing.

\section{Introduction}

The paper considers various control options for service mobile robots; the discovered approaches were also used in the industrial transport system (Industry 4.0) and in the process of creating an underwater walking vehicle with autonomous satellite robots. The operator in the most primitive approach can directly control the robot drives or monitor their operation in supervisory mode, using automatic obstacle avoidance by the control system, more complex level of algorithms - the operator gives the robot simple commands-synergy or sets the coordinates of the target point, 
or assigns the goal in a close to natural language. Three main functional blocks of the developed software that implement the listed modes: Planner, Navigator, and Pilot. Figure 1 shows the works developed by us in the weight category up to $30 \mathrm{~kg}$. In these robots, the blocks are located on Board the service robot or can be placed on computers remote via network channels, which act as a system controller with a graphical interface for the operator. The scheduler generates the target route after receiving the task from the operator, using a stationary model of the working environment from the robot database. This model contains metric, topological, and semantic information. The target route is formed as a sequence of sub-goals. Navigator, responsible for operational control of the robot's movement among moving objects in the environment based on input data - the target route, as well as on the readings of lidar and track odometers. The Navigator scans data and rebuilds the topological and metric models of the operational environment. While driving, this operating model is used to realign the local path, ensuring, that obstacles are eliminated. The local path and target route are formed from the list of sub-goals and passed to the pilot to control the drives.

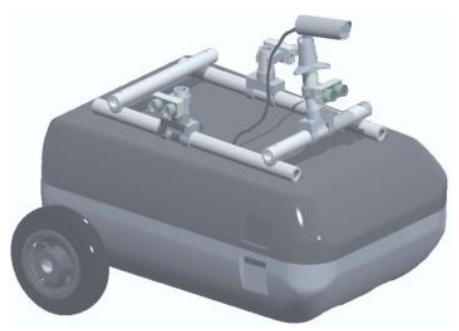

(a)

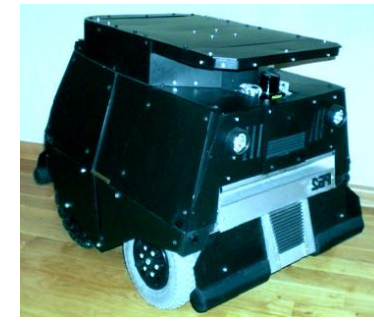

(b)

Fig. 1. Developed mobile robots AMUR-105 (a), RB-2 (b).

For the underwater walking vehicle, a control system was developed (figure 14), in which the Pilot unit is located on Board and connected by a cable with a control panel that has the functions of a Planner and Navigator (the functions of these units have the minimum necessary set of methods, but they are increased as the sensor capabilities of the device develop). This paper also discusses the proven technology of replication and intellectualization of the service mobile robot control, the study of the applicability of cycloidal speed reducer in the mechanical units of the AMUR-307 robot and the underwater walking robot. The developed mobile service robot AMUR-307 (Fig. 2) is designed for solving problems related to telemedicine, namely, moving objects between rooms. The robot has a crawler drive and a mounted customizable manipulator with two gripping devices, that the robot uses to interact with objects. During its development, special attention was paid to significantly reducing the time of replication and assembly of a group of robots due to the use of additive technologies, laser cutting technologies, the widespread use of plastics and ready-made mass production.
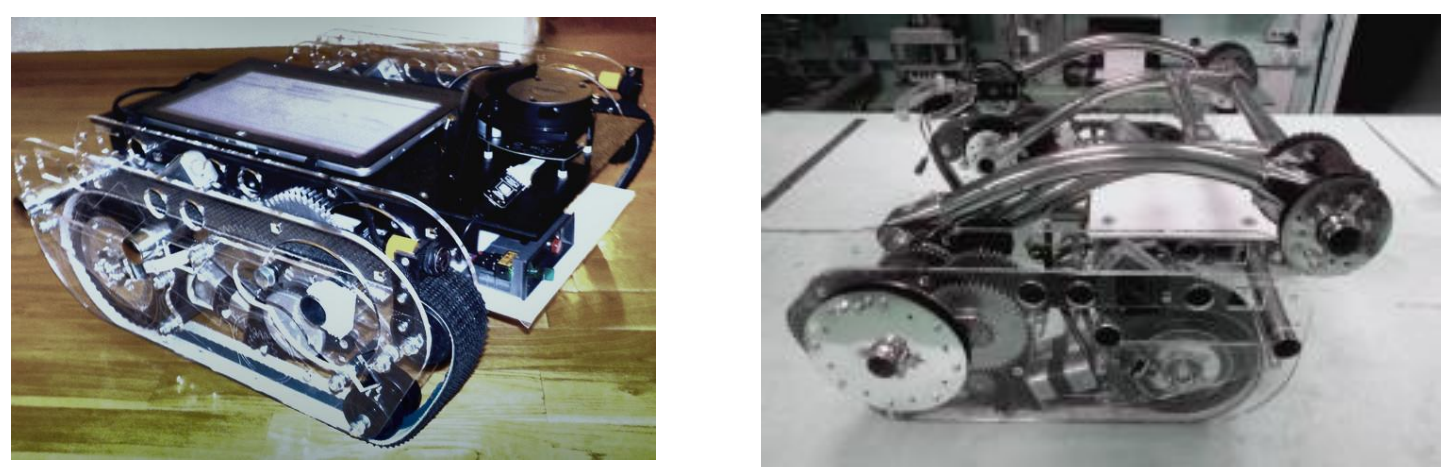

Fig. 2. Appearance of the AMUR-307 service robot, its own weight — up to $23 \mathrm{~kg}$.

The main tasks solved by such robots are the transportation of goods weighing up to $20 \mathrm{~kg}$ with the ability to open doors with standard rotary handles, capture objects, hold them and move them inside the premises and corridors of medical institutions as part of a comprehensive service. The robot also needs to be able to overcome ramps with a slopes of up to 35 degrees. One of the most important components of robotic devices is the Converter of the rotational motion of the drive to the reciprocating motion. The most common transmission used in modern gearboxes is the involute. This can be explained by a number of factors, such as: ease of manufacturing tools for cutting teeth, the presence of a large fleet of specialized equipment, a single technology for manufacturing and wheel control. In recent years, cycloidal speed reducers based on eccentric-cycloidal gearing have become widely used. This trend is due to a combination of several advantages of this gearing: compactness, smooth running, high power, reliability. The cycloidal speed reducer can reach a gear ratio of more than 150 for a single transmission. Eccentric-cycloidal gearing has an efficiency of up to $95 \%$, and at the same time almost $50 \%$ of the transmission teeth participate in gearing. 
At the same time, the specific mass of planetary gearboxes is less, than seven times in comparison with involute planetary gearboxes. In the cycloidal speed reducer, when the input shaft rotates with an eccentric mounted on it, an uneven rotation of the satellite is provided, which is connected directly to the eccentric. In these gearboxes, compensation mechanisms are usually additionally installed to eliminate uneven rotation of the output shaft. In this paper, we considered the applicability of such a gearbox with a significant simplification of the design (only a pair of eccentric-satellite parts).

To increase the torque and reduce the size of the structure of the manipulator of the Amur-307 service robot and the walking underwater vehicle, it is planned to use simplified cycloidal speed reducer made of polymer materials using laser and additive technologies. In accordance with this simplified gearbox manufacturing technology, the deviation of the output shaft path from the ideal circle is allowed. New type of transmission with output shaft mounted on an eccentric-cycloidal gearing satellite is proposed.

In the transport system of our workshop, the role of the Pilot is performed (shown in figure 3 ) by a multi-channel microprocessor power control board, and the Planner and Navigator are implemented on two computers of the technologist and operator, working in parallel to improve reliability. It is assumed, that the system automatically adjusts to different production scenarios and adapts to failures. The planner is a system of expert rules for moving 25 pieces of two - ton trolleys along 20 rail sections of tracks of various lengths with 5 cross lines-also rail tracks ("drivers") for rearranging trolleys between the main tracks. The Navigator analyzes the operation of about 40 sensors, that produce a series of several triggers, when trucks approach positions to change directions (on drivers). The corresponding program records the moments, when trucks enter the stopping zones, estimates their speed and calculates the braking moment for accurate fixation on the drivers. The main problem of building such programs based on the principles of Industry 4.0 is to identify potential sensor failures, synchronize the operation of drives (up to 60 units), and ensure, that trolleys are connected to the main equipment. To provide the necessary high frequencies of sensor polling ( 2 orders of magnitude higher, than the capabilities of industrial PLCs), a specialized microprocessor system was implemented, that preprocesses and collapses data to transmit it to the Navigator program on the operator's and technologist's control computers.
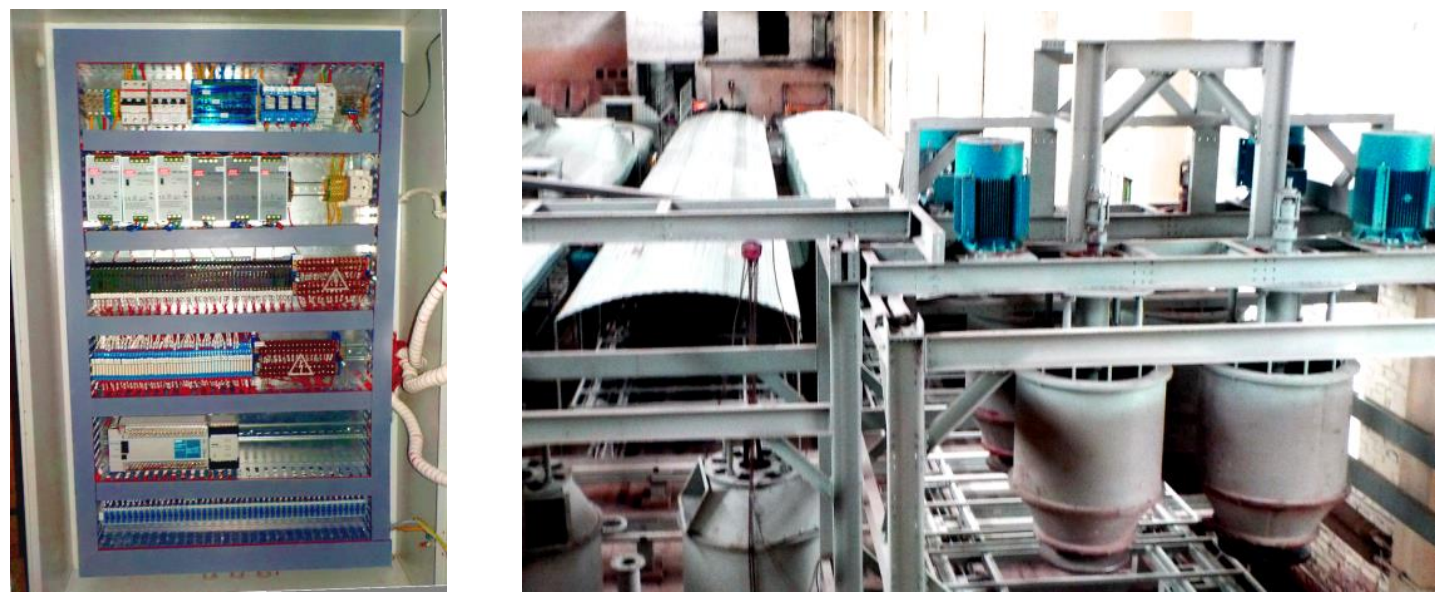

Fig. 3. Transport system control unit (left) for Industry-4.0

All these three areas of implementation of the developed approaches to control are united by the network principle of integrating multiple robots and mechatronic devices, which gives convenient access for the operator who control these multi-agent systems and has the ability to reprogram the control system. A corresponding integration software product has been developed [2], [40].

\section{Features of service control implementation}

The service robots considered in this paper were developed to work in the human environment, in urban apartments, in hospitals, schools, offices, and museums. These are TV surveillance robots, security robots, transport robots, and guide robots. According to their purpose, such robots should solve problems in the interests of humans, while moving confidently in closed rooms, surrounded by a small number of people. Unlike an industrial robot, a service robot needs to have a greater degree of autonomy and the ability to "understand" the environment and interact with people. A necessary condition for this is the intellectualization of control systems [19], [8], which solve the key task of ensuring movement in the operating environment. The motion control system under consideration was originally developed for service mobile robots RB-2 and Amur-105 with similar kinematics, with a wheel differential drive (shown in the left half of figure 1). In order to further develop algorithms and adapt to other robot mechanics, control system tests are conducted on the AMUR-307 crawler robot. 
The appearance of this robot for transport operations inside medical organizations is shown in figures 2 and 15 . the platforms of these robots are designed to accommodate the equipment needed to perform the service functions of robots. In the front part of the body of each robot, lidars are installed with scanning angles in the range from -140 to +140 degrees, limited only by the robot's structural elements. Odometers are installed on the wheel axles. The robots are equipped with video surveillance cameras. The robot's control system software is installed on onboard dual-core computers with a clock frequency of $1.8 \mathrm{GHz}$, running under the Windows 7 Embedded operating system, and there is an option for Linux OS. This low power is sufficient to implement full adaptive control in one of the modes listed below. The more functions the control system takes on, the less the human operator is involved in controlling the robot, and the more the person focuses on solving other important tasks. Let's distinguish six main modes, placing them according to the degree of complexity of the software:

1. Manual remote control mechanisms of the robot. The operator, via communication channels, remotely controls the robot's drives directly. This mode is the most common, the control system is extremely simple, and, generally speaking, there is no need for an on-Board computer. In the case of a robot with a differential drive, it is preferable to have an on-Board computing device for converting signals from the operator into drive control signals. For our Amur series robots and 4 robots (we upgraded them) for Ministry of Extreme Situations Russia, even this simple mode was used with software logical conversion of control signals in order to correct operator's errors by introducing dead zones, taking into account the dynamic capabilities and ergonomic features of the graphical interface for the operator.

2. Command remote control of the robot's movement. The operator gets information about the operating environment and controls the robot using commands. The coordinates of the target point can be set relative to the current position of the mobile robot by specifying the azimuth and distance. The implementation of this control method is no longer possible without a computer on Board the robot. The function of providing obstacle avoidance lies with the person, as well as intervention in emergency situations.

3. Route-remote control of the robot's movement. This mode differs from command-remote mode in that a preprepared route and coordinates of sub-goals are transmitted for execution, which the robot passes as a single command. The person tracks the detour of obstacles, that arise in the path of the robot's movement.

4. Route-supervisory control of the robot's movement. This mode differs from the route-remote mode by the presence of environmental assessment sensors and an automatic obstacle avoidance program on Board. The operator only monitors the execution of the route.

5. Coordinate-target supervisory control of robot movement. The operator determines the coordinates of the target point of movement, and the route, in the form of a chain of sub-goals, builds the upper level of the control system - the Planner. The control system must have a metric or topological-metric model of the operating environment [20], as well as algorithms for finding the path from the current position of the robot to the target position. The operator only observes and intervenes in control process in emergency situations.

6. Semantic-target supervisory control of robot movement. This mode differs in that the operator formulates the move goal as the object name in quasi - natural language. The planner independently identifies the location of the target object and builds a route. To do this, the Scheduler must have a topological-metric model and a semantic model of the operating environment associated with it [21]. The semantic model must contain the names of all objects in the topological-metric model, their properties, relationships between them, and rules for logical inference. The planner implements inference rules for constructing and semantic route descriptions based on information from the database, that is part of the traffic control system (the operating environment model for RB-2 and AMUR-307). Then, using the topological-metric model, the semantic description of the route is translated into a sequence of coordinates of sub-goals.

All supervisory modes (from the 4th to the 6th) increase the robot's autonomy and adaptability, but to implement these modes, adequate obstacle sensors must be installed on Board (on our robots, these are lidars and IR sensors). For implementation, you must also create an operating environment model in advance. Planners working with topologicalmetric and semantic models actually implement elements of artificial intelligence. The RB-2 control systems and the Amur-307 series of robots operate in all these modes, and the changeover from one mode to another is carried out only by changing a small set of parameters.

\section{Architecture of the developed control system}

The architecture of the RB-2 and AMUR-307 robot motion control system is shown in figure 4. the control System has four main blocks: a Monitor, a Planner, a Navigator, and a Pilot. All blocks of the system use a data loop that stores the history of the robot's movement, data generated by the Navigator and other blocks.

The Monitor control the work of all levels and receives tasks from a operator, and if they are not completed, requests additional information, starts or stops processes. At the first launch, the monitor initiates the configuration of the control system, forms functional restrictions. The algorithm of its operation is based on a system of expert rules, that exclude breakdowns and harm from the robot's actions [22].

The Scheduler is initiated by the Monitor in coordinate-target or semantic-target supervisory control mode. Its task is to build a target route [22] based on a stationary model of the operating environment. The planner builds a route before the robot starts moving. The control system allows you to re-plan the route while driving, for example, at the request of the operator. 


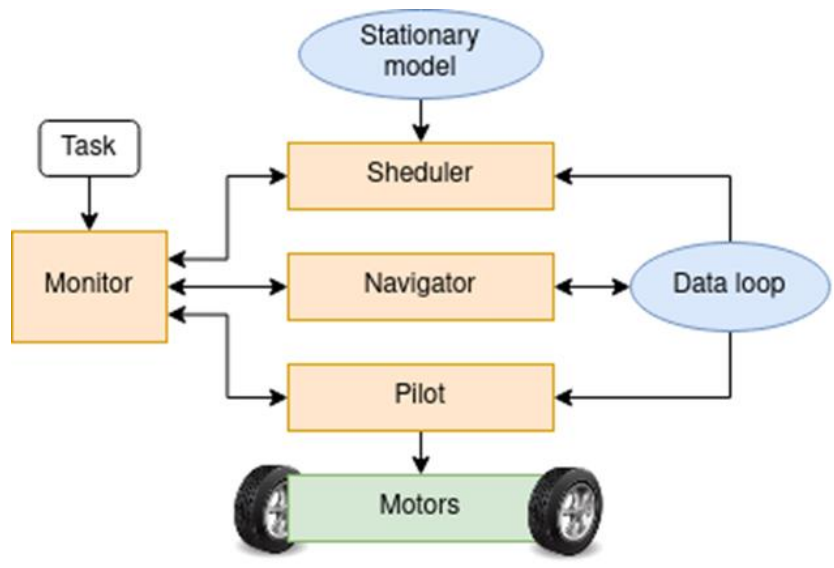

Fig. 4. Architecture of the control system of the developed service robots

The Navigator provides safe control in the environment of external moving objects based on data from lidar. An operational model is constructed, which is topological-metric and is used by the obstacle avoidance algorithm to build a local path from the current robot position to the most remote sub-goal located in the "field of view" of the scanner with an obstacle envelope. During each scan cycle, the operational model is completely rebuilt, and an updated local path is formed, including information from odometers. All this data is immediately recorded in the loop. If the passage to the target position is completely blocked, the Navigator sends a corresponding signal to the Monitor and stops its work, notifying the operator of the failure.

The Pilot, driving a differential drive, the robot leads to the next sub-goal local path using the method of minimizing positional and angular error (deviation of the direction of the velocity vector and the longitudinal axis of the robot from the direction to the subgoal), the algorithm published in [23], [24]. At the same time, the "comfortable" driving mode must be observed, which does not create an overload in the power supply circuits of the engines. The pilot updates the control signals to the drives immediately after entering the next data in the loop (after updating the data about the robot's position).

\section{Solving problems of planning target routes}

The target route is formed as a sequence of sub-goals. Each sub-goal is constructed as segments (gates and passageways) that the robot must cross in the specified direction. The minimum width of the passage is determined with a margin on each side due to the permissible positioning error (for RB-1 it is $62 \mathrm{~cm}$, and for AMUR-307 - $68 \mathrm{~cm}$ ). Planning takes place on the generated stationary model of the environment [25], entered in the control system database [26], [27]. All static objects of the operating environment are obstacles and are therefore excluded from the area of possible movement, from the working area approximated by a non-convex polygon. This area is automatically divided into convex polygons - passframes, that are free of obstacles. Passframes composed from arrays of vectors are ordered counter-clockwise direction, and marker signs. The procedure for their construction is described in detail in [28]. Network Passframes forms topology-the metric of a stationary model of the workspace of the robot. Pairs of adjacent Passframes are connected to each other if they have the same vectors directed in opposite directions, this is fixed by mutual references in the corresponding lists. Figure 5 shows a fragment of an office space (covered by a network of Passframes) - a graphical representation of a stationary model (a map of the operating environment). Point A is the robot's starting position, and B is the target position set by the operator. Sub-goals $\mathrm{C} 1, \ldots$, and $\mathrm{C} 4$ are generated at the midpoints of Passframe junctions by direct search of network nodes in depth with returns and checking for cyclicity, from A to goal B [29].

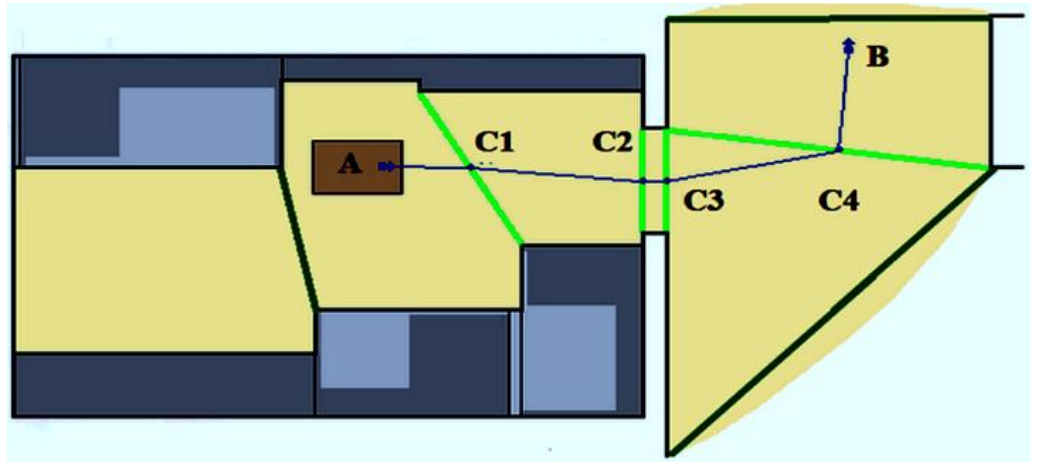

Fig. 5. Passframe network and target route. 
In semantic-target control, the goal of the robot's movement is set as the name of the target object. The scheduler must have a semantic model of the operating environment with references to Passframe IDs in the topological-metric model. This model contains sets of predicate expressions and logical relations between phenomena, objects, and properties [30], [33]. The model includes: Terms, Predicates, Statements, Rules, and Scenarios. The Russian version of the STRIPS language is used to describe the model [31]. Semantic expression processing, logical inference, and scheduling are performed by the Scheduler based on the classical Graphplan algorithm [32]. For a service robot, the semantic model is important not so much for controlling movement, but for providing a dialogue with the operator and forming a robot behaviour plan, which includes objects for manipulation in addition to the room plan [34]. In [2], [38], [39], [40], other options for implementing the scheduler are also considered.

The robot's movement safety should be based not only on static (a priori) data about the operating environment, but also on operational information from the sensor system and lidar (for more information, see [35], [36], [37]). The Navigator constantly recreates the operational model of the environment, on the basis of which the local path is rebuilt, which ensures, that the robot evades dynamic and unaccounted-for obstacles along the way. The duration of this update cycle is from 100 to $145 \mathrm{msec}$. For used scanners, the control area is limited to a radius of 3.5 meters. This data is compared with odometer readings updated every $33 \mathrm{msec}$. All this information, along with timestamps, is stored in the loop. The structure of Passframes operating model and Passframes static models are identical.

Data from the laser scanner - an array of distances to obstacles in the polar coordinate system, is processed by a modified Split-and-merge algorithm [38], for extracting straight line segments, that limit the area free of obstacles (shown in yellow in figure 6. It can be seen that the stationary model diverges from obstacles in the scanner's "visibility" zone, and the zone is approximated by a network of Passframes [28]. Several faces of Passframes are located outside the" visibility " of the robot, such borders are indeterminate and it is likely that there is free space for the robot to move.

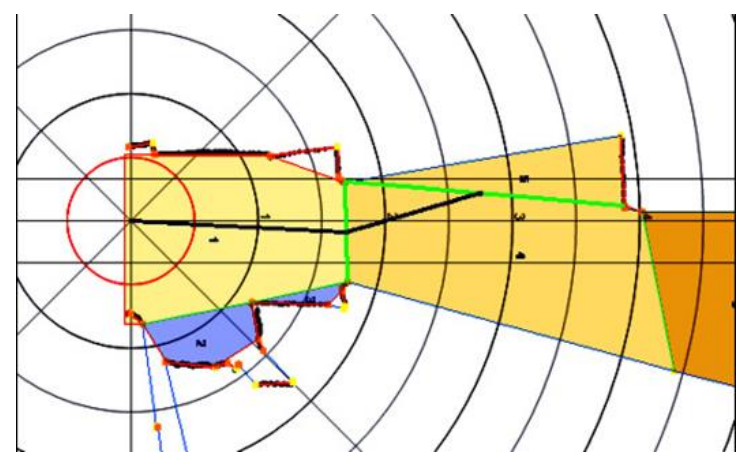

Fig. 6. Generation network Passphrases operational model according to the lidar

The operational model is used to build a local path, that connects the current position of the robot with the next actual sub-goal of the route, skirting obstacles in the way of movement. The local path is cyclically rebuilt with the frequency at which the lidar generates information. Just like the target route, the local path is formed as a sequence of sub-goals, each of which represents an passage for the robot. The algorithm for constructing a local path is reduced to the problem of searching for a chain of Passframes, which is solved by the method of direct search of network nodes in depth, with returns and checking for cyclicity [29]. For each selected node in the network selects a neighboring node Passframe, that previous Passframe has a common border pass between them. A chain of Passframes is formed, and the midpoints of the passes between them become sub-targets of the local path, that is sent for execution.

If the robot does not have a laser scanner, the Navigator selects the remaining section of the target route as the local path. For safe driving, the operator will have to actively participate in control, quickly respond to obstacles, that arise in the way of movement, form control signals, avoiding collisions. An effective option is the use of ultrasonic sonars, taking into account their wide radiation patterns. Even when surrounded by a small number of people, this requires constant attention and distracts the operator from solving other equally important tasks. This mode can be used, for example, for night security robots moving in a static operating environment, in deserted rooms.

\section{Integration software and network access}

The development of another top - level add-on-integration, distributed software, which also includes 3D simulators (digital clones), that work in real time in parallel with real robots, became the basis for building control of the grouping of intelligent mobile robots considered above, united in robotariums. Currently, almost two dozen robots and industrial stands, cameras for remote inspection of robotariums are used for research. The created distributed research and experimental laboratory is actively used for research, training and implementation purposes under the project"Intelligent robotronics". Due to the unification of the integration process of different types of mechatronic devices and the construction of appropriate software, the implementation time of sensor-control systems and intelligent algorithms, including those based on deductive mechanisms, has been radically reduced. 
The created models of the robot environment make it possible to automatically form trajectories of evasion from moving and stationary obstacles based on data from lidars, ultrasonic and cameras, including using parallel calculations [39]. The proposed programming technologies made it possible to speed up the process of developing new equipment due to distributed access and special organization of interaction between software components. The use of multidimensional logic allowed us to intellectualize the work of distributed groups of robots in extreme conditions, thereby removing the load from the operator. The developed principles of controlling mechatronic device complexes have been applied in creating remote control of complex production being rebuilt according to the Industry 4.0 type and for underwater walking apparatus [2], [8], [40]. Network software for remote control of robotariums with a large number of robots and devices allows several operators/students/developers to simultaneously have access via VPN channels not only to robots, but also to their individual components, including using digital counterparts of real robots for pre-debugging control system programs or for reprogramming robots. Naturally, such broad opportunities required the use of organizational and programmatic measures to protect against unauthorized or unqualified access. Currently, access levels are implemented from the student to the developer with appropriate data protection. The use of digital doubles also allows you to use them as a supplement to the work of real cameras, to observe invisible angles, which significantly expands the possibilities of controlling and debugging intelligent algorithms (figure 7). Various aspects of our project "Intelligent robotronics" are discussed in [8], [19], [20], [21].

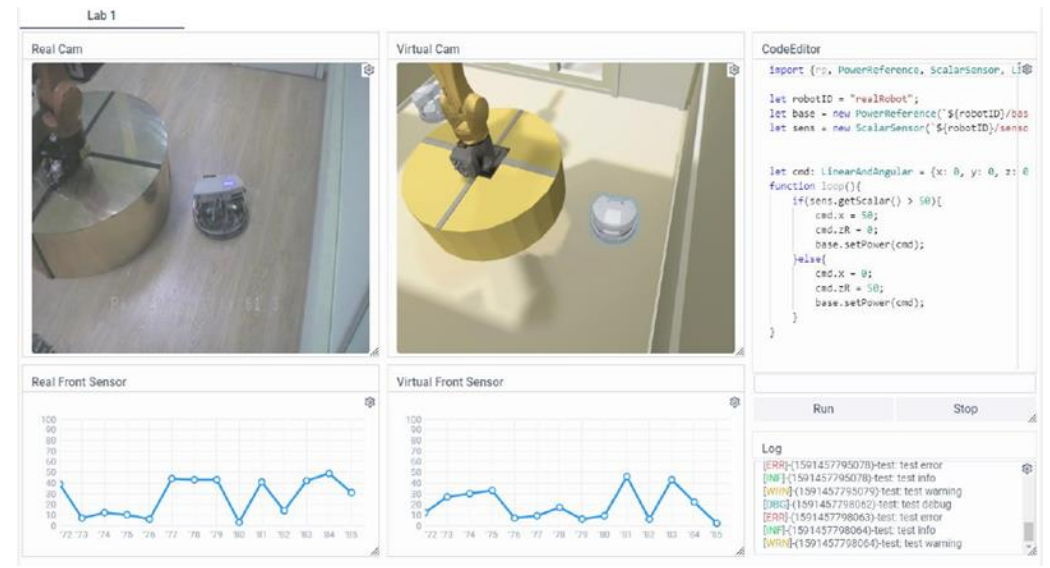

Fig. 7. The digital double (clone) of the robotarium makes it possible to change the viewing angle.

For example, one of the developed algorithms is a program for local execution of mobile robot movement missions between set goals (including between rooms) with automatic obstacle avoidance. This implemented software package is based on the proposed technology for generating Passframes based on rangefinder data from a circular lidar. The safety system is provided by infrared and contact bumpers, as well as ultrasonic sensors. The path is calculated using odometers and their readings are reset when passing markers. Mission planning work is performed using a parallel processor. Field and computational experiments have shown the effectiveness of the proposed algorithms for intelligent robot control and the possibility of their application in various environmental conditions, including hospital-type premises (subject to appropriate settings for specific types of missions).

\section{Design of the electronic hardware of the AMUR-307 robot}

Due to the large number of sensors and controlled electromechanical de vices, the presence of a FullHD camera requires the use of an on-Board computer. Among those considered were systems built on ARM and x86 processors. Due to lower power consumption, preference was given to the ARM processor family (Raspberry Pi, Orange Pi, Rock960, Jetson Nano, and others). Further, the choice was made based on such indicators as power consumption, availability of GPIO pins, computer cost, the number of libraries and frameworks for these computers, as well as the quality of support from the manufacturer or from the developer community. As a result of comparative research, the Raspberry Pi 3 b+ turned out to be the best for use in this project, since this Board has: 4 productive Cortex-A53 cores with a fairly low power consumption (up to $15 \mathrm{~W}$ ), a unified GPIO connector and convenient standard dimensions of the Board itself, which in turn allows you to use many other compatible boards. This microcomputer also has a large number of libraries, frameworks, operating systems, and various ready-made projects, written by one of the largest communities of microcomputer programmers.

AMUR-307 has six collector motors, the maximum current consumption of each of the engines is $15 \mathrm{~A}$. To control these motors, motor drivers with an external PWM signal were considered. Among all the considered power switches, VNH2SP30 was chosen, since they have 2 channels per chip at a maximum current of 30A and an external PWM signal for each channel. They are delivered in the form of Arduino-modules with binding and 2 VNH2SP30 chips, which in turn allows you to control 2 motors with the necessary accuracy. One of the key elements of the control system is the developed cross-Board. A prototype of the cross-Board was made and its operating modes were investigated. 


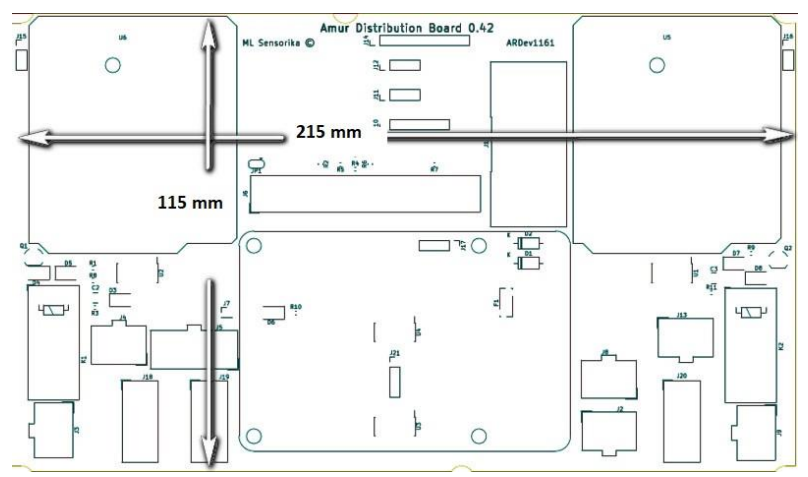

Fig. 8. layout of seats for electronic components and modules of the designed cross-Board.

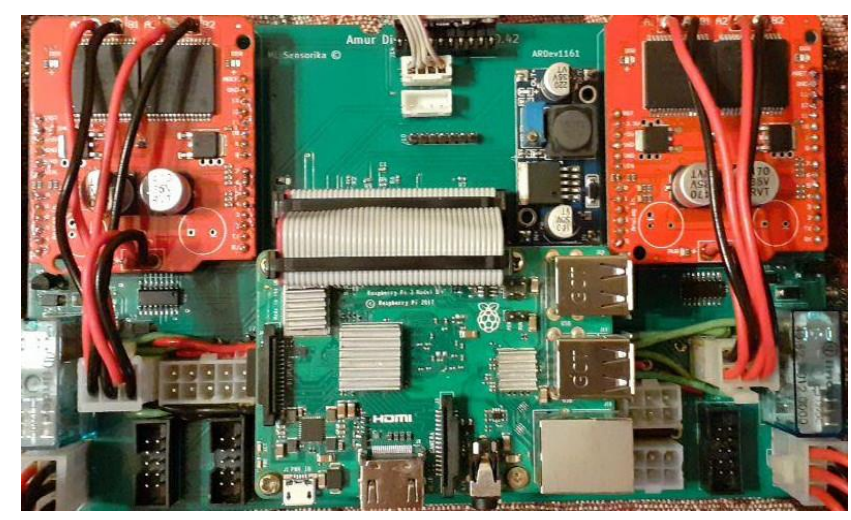

Fig. 9. the developed Board Assembly with modules is mounted in a semi-hermetic case $80 \times 125 \times 225 \mathrm{~mm}$.

Based on the results obtained, the main version of the cross-Board was created with additions and fixes to the functionality. It was developed and tested using KiCAD CAD software, and manufactured at the Resonit plant (figures 8-9). It was possible to create a powerful small-sized and fairly universal control unit, which was used for further development and configuration of the software. Replicated boards were manufactured using surface mounting technology, which allowed to increase the density of the layout and reduce the cost of production. The Board is twolayer, both layers are covered with polygons connected to the ground, which reduces high-frequency interference from PWM modulation of power keys.

\section{Development of the onboard system software}

For remote interaction with the robot, you must use a client-server architecture that enables add more and adjust onBoard algorithms, for example, to return the robot to the point with a steady Wi-Fi signal in case of ties. The created system uses the following open source software (figure 10):

GStreamer - broadcasting an H.264 encoded video stream;

Protocol Buffers - mechanism for serialization/deserialization of control data and sensor signals;

$\boldsymbol{g R P C}$ wrapper for Protobuf for more flexible data control;

Open $\boldsymbol{C V}$ - computer vision algorithms for processing a video stream on the server;

WiringPi is a library of algorithms for interacting with Raspberry Pi GPIO outputs.

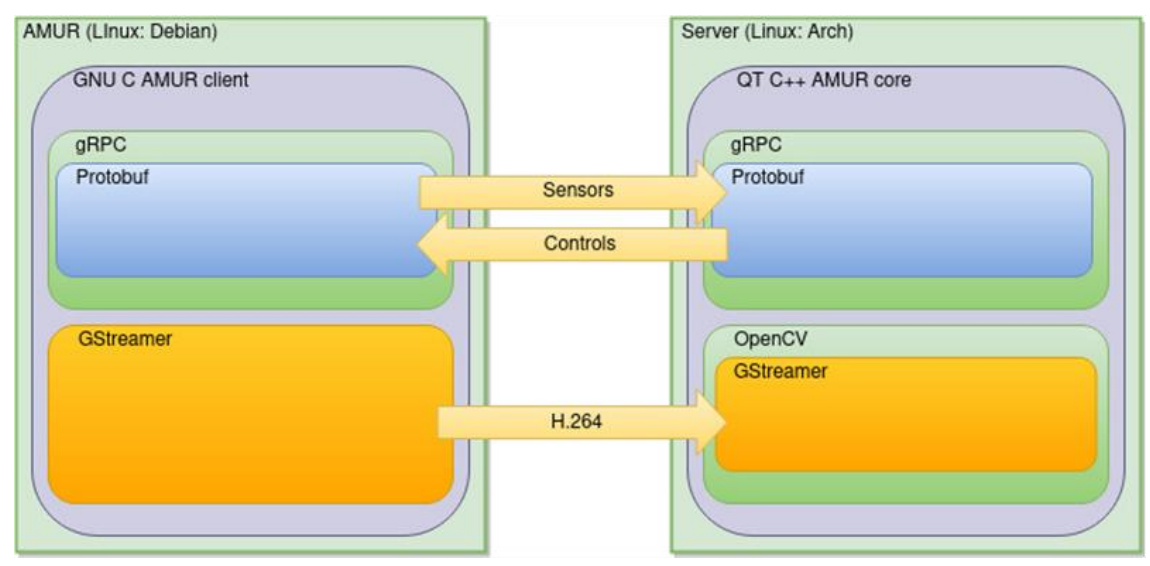

Fig. 10. Client-server interaction diagram of software components.

The developed software uses an object-oriented programming paradigm to improve code readability and isolation of objects from each other. Client software has a microservice approach. The main class of the application uses the LogicController microservice, which hides interaction with the network, peripheral hardware, and internal hardware capabilities of the Raspberry Pi and has additional algorithms for processing data received from lower-level objects. The LogicController class uses the PeripheralController microservice, which receives and preprocesses data coming from all external sensors, and also transmits all control data to external devices, such as motors, servos, LEDs, and so on. LogicController uses the child microservice NetworkController, which in turn encapsulates the interaction mechanisms for working with the TCPClient network client class. LogicController also has a Timer class for calling functions for transmitting data over the network and tracking the time when electric motors are switched on. 
The PeripheralController class interacts with the RegisterController class, which provides mechanisms for interacting with cross-Board shift registers through child classes. The HC595 child class provides an interface for interacting with $74 \mathrm{hc5} 55$ shift registers, which are used on the cross-Board to expand output ports, and HC165 with $74 \mathrm{hc} 165$ registers to expand input ports. Peripheral Controller uses the Peripheral Settings class, which stores and allows you to control peripheral settings to change the settings of future boards and backward compatibility with previous ones. The settings in the Peripheral Settings class are divided into groups and are also encapsulated in the corresponding classes, such as PWMPins, HC595Pins, and HC165Pins. The Peripheral Controller class also has a child PWM Controller microservice, which encapsulates the logic for controlling Raspberry Pi PWM channels.

\section{Study of the applicability of cycloidal speed reducer}

The aim of the study was to analyse the possibility of using cycloidal speed reducer is a new-type to rotate the lower links lifting and gripping mechanism (manipulator) service robot AMUR-307 and the analysis of possibility of application of cycloidal speed reducer new type to convert the rotary motion into reciprocating, for example, underwater walking robot, using the Chebyshev's Lambda Mechanism. The study continues previous work [5], [8], [10], [11], [12] and is applicable to many types of robots, for example, for the one presented in [13]. More detailed results of research on a walking drive using a modified Chebyshev mechanism can be found in the works of Volgograd state technical University [14], [15], [16], [17], [18]. First of all, we studied the trajectory of the output link fixed on the satellite of an eccentric-cycloidal gearing with a gear ratio of 1:20 using the graphical method. The resulting path of the output link is shown in figure 11, where D is the ideal trajectory of rotation of the output link; A - the deviation of the trajectory endpoints output link cycloidal gear from the circle; $\varphi$ is the discrete rotation angle of the output link (step angle adopted for the calculations).

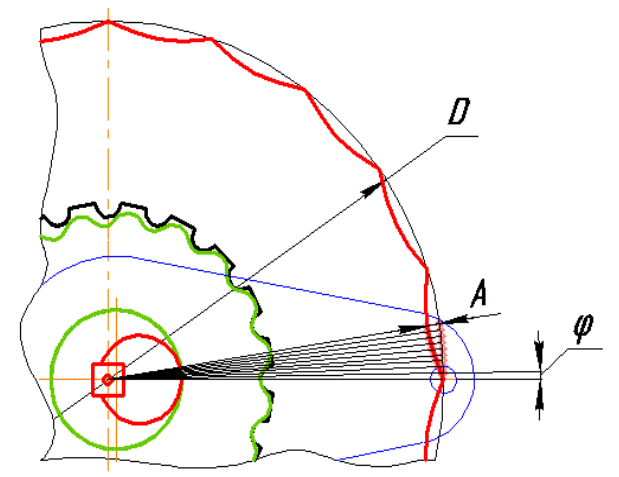

Fig. 11. Diagram of the trajectory of the output link of an eccentric-cycloidal gearing with a gear ratio of 1:20

We introduce the value $\Delta$ to estimate the relative deviation of the trajectory of the output link of an eccentriccycloidal engagement from the ideal trajectory:

$$
\Delta=\frac{A}{D} \cdot 100 \%
$$

The relative deviation of the path $\Delta$, when the output shaft is rotated by an angle from $0^{\circ}$ to $9^{\circ}$ is shown in figure 12 . With the considered design parameters of the gearbox, this deviation is maximum at an angle of $9^{\circ}$.

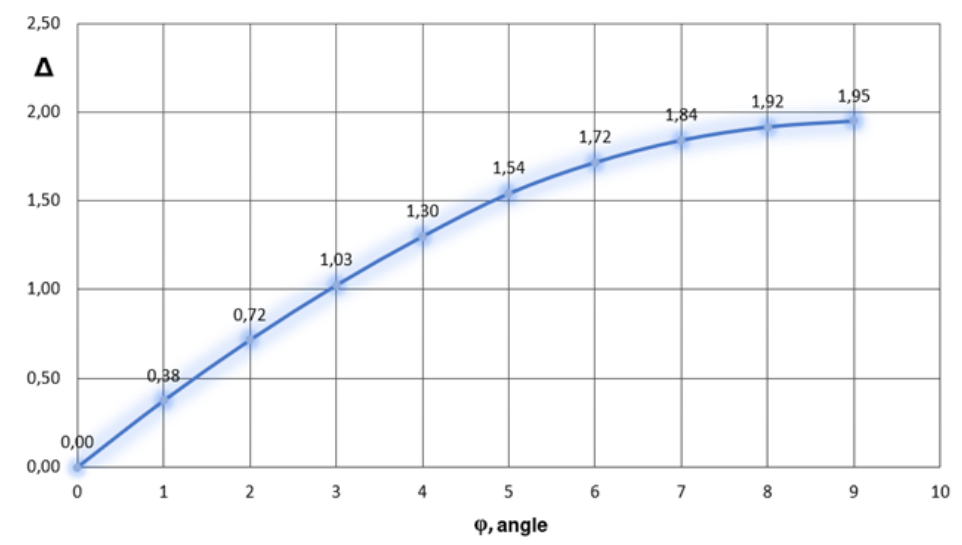

Fig. 12. Graph of changes in the relative deviation of the cycloidal engagement path $\Delta, \%$ of the desired circle 
Using a CAD system, it was possible to analyze the animation of the walking thruster and construct the trajectory of the output link of the Chebyshev's Lambda Mechanism connected to the feet of the walking underwater robot, using a gear with an eccentric-cycloidal gearing as the drive of the input link of the mechanism. Figure 13 shows two step cycle trajectories for the two main types of gaits: with a drive link (crank) from a conventional gearbox (circular movement) and with a drive from a simplified cycloidal speed reducer (the trajectories are shown in a bolder line), where nonsmooth areas (peaks and troughs) are faintly visible.

$$
a=45^{\circ}
$$

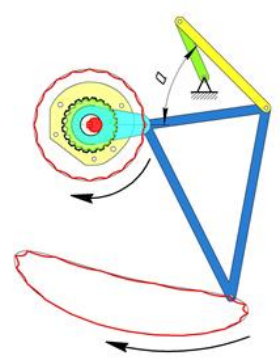

$a=30^{\circ}$

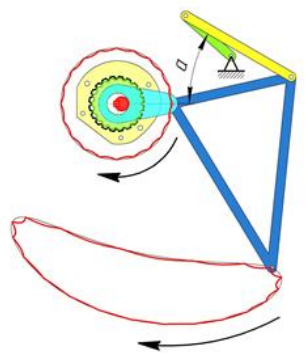

$a=15^{\circ}$

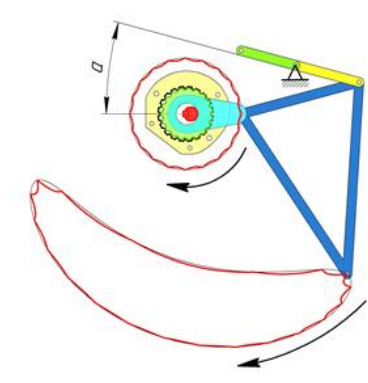

Fig. 13. The trajectory of the foot of the walking device relative to the body (step cycles) at different angular positions of the rotary lever $(\beta)$, which determines: a) the main marching gait at $\beta=45^{\circ} ;$ b) the gait for stepping over high obstacles at $\beta=15^{\circ}$ and in betwean.

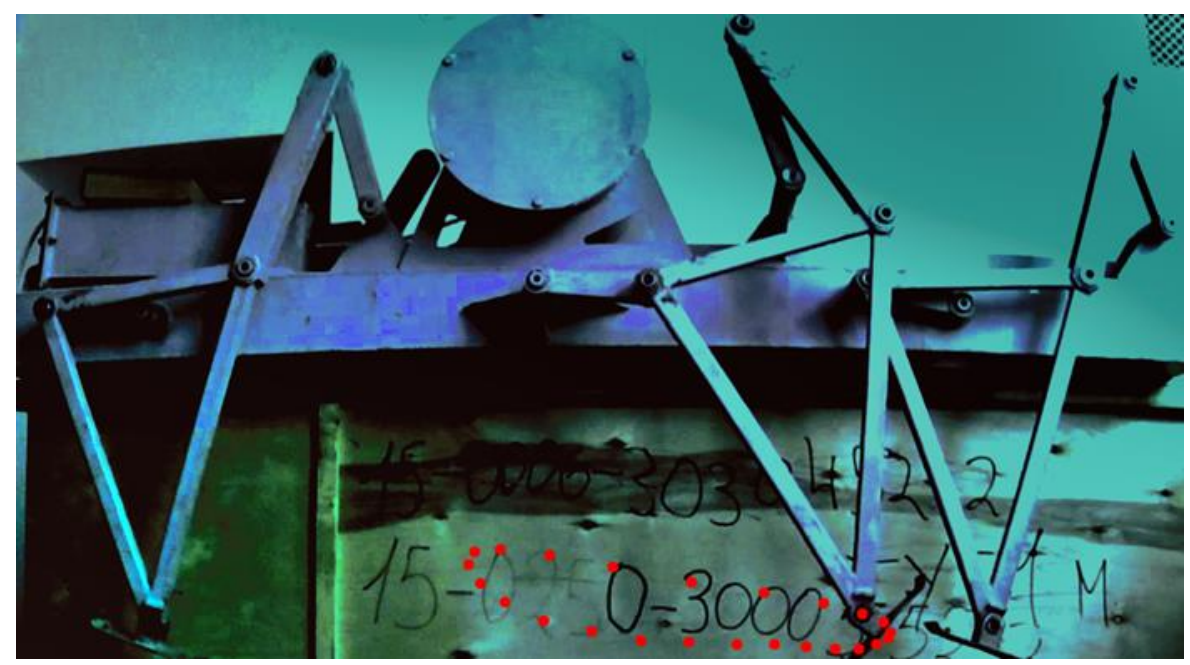

Fig. 14. Reference trajectory of the foot of an underwater walking vehicle

Figure 14 shows a kinogram of the actual movement of the foot of the walking mechanism. The points on the trajectory correspond to the position of the foot when the drive crank is rotated by a fixed discrete angle. With this trajectory, the work of the walking mechanism was compared with the standard gearbox and with the new cycloidal speed reducer (compare figures 13-14).

\section{Conclusion}

The current stage of this project in terms of creating onboard mobile robot systems is the construction of several samples of service mobile robots based on the developed rapid prototyping technology and their production using laser technologies. Development and testing of the motion control system was carried out, the composition of the onboard equipment and the structure of the software are being worked out. The features of controlling robots with both wheeled and tracked drive were studied. Control was realized in remote and supervisory modes, with or without a stationary model (figure 15). The tests were conducted in an office space and showed, that the system architecture, operating environment models used, and software algorithms described in this paper are highly efficient. Depending on the composition of the onboard equipment and the state of the external environment, the system can be easily rebuilt without losing its functionality. The speed of the software allows you to control the movement of robots in real time, even with onboard computers, that are not of the highest performance. The network approaches used significantly expand the application areas of the developed mobile service robots and robotariums. 

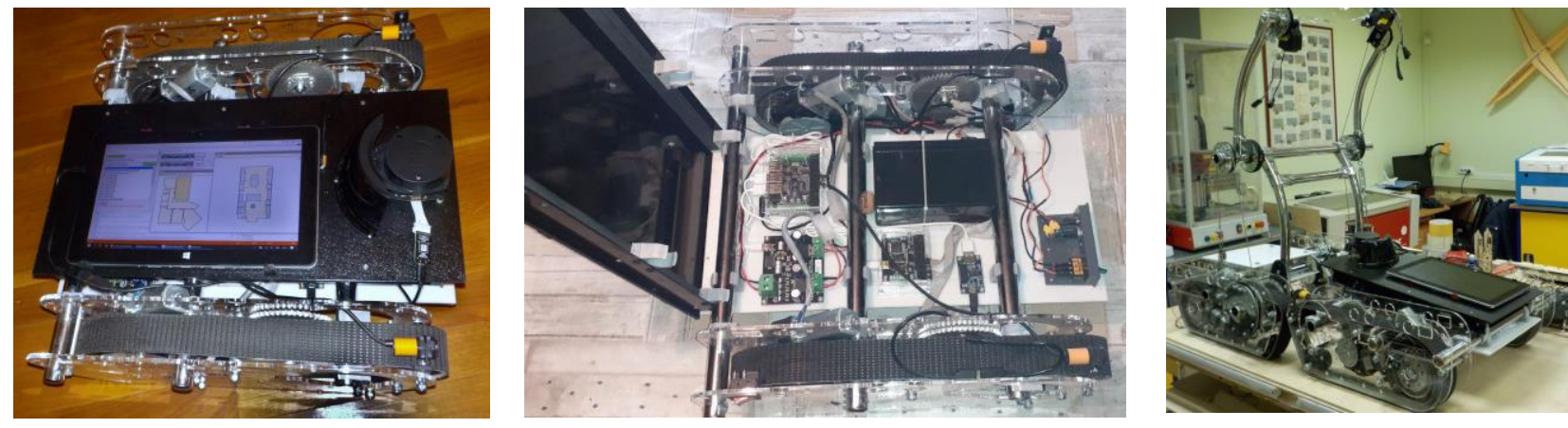

Fig. 15. Displaying data from the leader on Board of the robot (left). Development of technology for replication of service robots Amur-307

As a result of research and development work, a cross-board was designed, developed and manufactured to connect the computing device to all sensors and electromechanical devices of the robot. Compact microprocessor units for multi-channel control and sensor data acquisition was installed. Client software was developed for the AMUR-307 service robot, which provides a network interface for interacting with all robot devices, as well as network software for managing robotariums. Full-scale and computational/virtual tests of the software and hardware environment for controlling the AMUR series robots and the proposed algorithms for intellectualizing control in robotariums were carried out. The applicability of cycloidal speed reducer showed, that the relative error deviations of the trajectory endpoints output link of the mechanism of eccentric-cycloidal gearing compared to the trajectory of a perfect circle is within only some percents. This makes it possible to use such a gearbox with a simplified scheme of the cycloidal speed reducer in the lifting and gripping device of the Amur-307 mobile service robot. In this case, an elastic element (elastic compensating coupling) is additionally installed between the corresponding link of the manipulator and the planetary-tipping mechanism.

The use of a mechanism with an eccentric-cycloidal gearing as a drive for the input link of the walking device showed that in the reference part of the walking path, deviations from the "smooth trajectory" are minimal (no more than $2.5 \%$ ), such deviations are insignificant for the implementation of walking, visually indistinguishable. At the same time, the design of the walking device is significantly simplified and the cost of the product is reduced.

\section{References}

[1] Pryanichnikov V.E., Aryskin A.A., Eprikov S.R., Kirsanov K.B., Khelemendik R.V., Ksenzenko A.Ya., Prysev E.A., Travushkin A. S. (2017). Technology of Multi-Agent Control for Industrial Automation with Logical Processing of Contradictions, Proceedings of the 28th DAAAM International Symposium, pp.1202-1207, B. Katalinic (Ed.), Published by DAAAM International, ISBN 978-3-902734-11-2, ISSN 1726-9679, Vienna, Austria. DOI:10.2507/28th.daaam.proceedings. 167

[2] B. Katalinich., A.Ya. Ksenzenko, S.V. Kuvshinov, Yu.S. Marzanov, E.A. Prysev, V.E. Pryanichnikov, R.V. Khelemendik, S.R. Epricov (2016)// Development of distributed software-hardware robotarium. Extreme robotics. // Proceedings of the International scientific and technical conference. (November 24-25, 2016, St-Petersburg) Saint Petersburg: OOO"AP4Print", 2016. - 480 p., P.459-465. ISBN 978-5-9909163-3-3. Extreme robotics. // Abstracts of the International Scientific and Technological Conference. - Saint-Petersburg: "Gangut", 2017. - 272p. URL: http://er.rtc.ru/images/docs/Sbornik_tezisov_ER_2017.pdf

[3] Pryanichnikov V.E., Ksenzenko A.Ya., Kuvshinov S. V., Poduraev Yu. V., Prysev E.A., Khelemendik R.V., Eprikov S. (2016). Intelligent robotronics: hardware-software complexes of robotariums, Proceedings of the 27th DAAAM International Symposium, pp.0225-0229, B. Katalinic (Ed.), Published by DAAAM International, ISBN 978-3-902734-08-2, ISSN 1726-9679, Vienna, Austria DOI: 10.2507/27th.daaam.proceedings.033

[4] Bogdanovich A.V., Kirsanov K.B., Pryanichnikov V.E., Khelemendik R.V. (2018). Hardware-Software components of intelligent service mobile robots / Information-measuring and control systems (Intelligent and adaptive robots, vol. 14, № 1-2, 2019), M.: Radiotechnika, 2018, vol. 16, № 12, p. 33-39.

[5] Bogdanovich A.V., Kirsanov K.B., Khelemendik R.V., Pryanichnikov V.E., Prysev E.A. (2018). Development of Technology for Manufacturing Hardware and Intelligent Levels of the Service Autonomous Mobile Robot AMUR-307, Proceedings of the 29th DAAAM International Symposium, pp.1313-1318, B. Katalinic (Ed.), Published by DAAAM International, ISBN 978-3-902734-20-4, ISSN 1726-9679, Vienna, Austria DOI: 10.2507/29th.daaam.proceedings.189

[6] Aryskin, A[lexander]; Bogdanovich, A[lena]; Davydov, O[leg]; Grigoriev, A[leksey]; Khelemendik, R[oman]; Kharin, K[onstantin]; Kuvshinov, S[ergey]; Petrakov, M[aksim]; Plotnikov A[leksey], Pryanichnikov,

V[alentin]; \& Tarasov R[adomir] (2019). Control algorithms for service and industrial transport robots, Proceedings of the 30th DAAAM International Symposium, pp.1166-1173, B. Katalinic (Ed.), Published by 
DAAAM International, ISBN 978-3-902734-22-

8, ISSN 1726-9679, Vienna, Austria.

DOI: 10.2507/30th.daaam.proceedings.164

[7] Aryskin, A[leksander] A[.]; Bogdanovich, A[lena]; Davydov, O[leg]; Khelemendik, R[oman]; Petrakov, M[axim] \& Pryanichnikov, V[alentin] (2019). Control and Interaction Algorithms for Industrial and Service Robots, Chapter 33 in DAAAM International Scientific Book 2019, pp.377-384, B. Katalinic (Ed.), Published by DAAAM International, ISBN 978-3-902734-24-2, ISSN 1726-9687, Vienna, Austria DOI: 10.2507/daaam.scibook.2019.33

[8] Pryanichnikov V.E., Chernyshev V.V., Arykantsev V.V., Aryskin A.A., Eprikov S.R., Ksenzenko A.Ya., Petrakov M.S. (2018). Enhancing the Functionality of the Groups of Autonomous Underwater Robots, Proceedings of the 29th DAAAM International Symposium, pp.1319-1325, B. Katalinic (Ed.), Published by DAAAM International, ISBN 978-3-902734-20-4, ISSN 1726-9679, Vienna, Austria DOI: 10.2507/29th.daaam.proceedings. 190.

[9] Tarasov R. B. (2019)/ Development of the hardware and software part of the tracked service robot AMUR-307 // materials of the V all - Russian student conference (Yoshkar-Ola, November 5, 2019): at 8 parts. Part 3: New technologies of Infocommunications, radio engineering and electronics for breakthrough industries. -Yoshkar-Ola: Volga state technological University, 2019. -138-142 pp. ISBN 978-5-8158-2133-0 ISBN 978-5-8158-2136-1 (Part 3)

[10] Solovyova M. D., Shchepin V. D. (2016)/ Improvement of the technological process of manufacturing cycloidal speed reducer (PCR) using blade Processing methods // Scientific progress - creativity of young people: materials of the XI international youth scientific conference on natural science and technical disciplines (Yoshkar-Ola, April 22-23, 2016): at 4 o'clock / ed.: D. V. Ivanov [et al.]. - Yoshkar-Ola: Volga state technological University, 2016. Part 2. -264 p.

[11] V.V. Chernyshev, V.V. Arykantsev, A.E. Gavrilov (2016), Design and underwater tests of subsea walking hexapod MAK-1, in Proc. of the ASME 2016 35th Int. Conf. on Ocean, Offshore and Arctic Engineering (OMAE 2016), (Busan, Korea, 2016).

[12] V.V. Chernyshev, V.V. Arykantsev, Ya.V. Kalinin (2017), "Underwater Tests of The Walking Robot MAK-1”, Human-Centric Robotics. Proceedings of the 20th International Conference on CLAWAR 2017, Professional Engineering Publishing, 2017, pp. 571-578.

[13] S.Y. Yoo, B.H. Jun, H. Shim (2014), Design of static gait algorithm for hexapod subsea walking robot: Crabster, Transactions of the Korean Society of Mechanical Engineers A, Vol. 38, Issue 9, pp. 989-997.

[14] Chernyshev, V.V. (2014) / Traction properties of walking machines on underwater soils with a low bearing ability / V.V. Chernyshev, A.E. Gavrilov // Minerals of the Ocean - 7 \& Deep-Sea Minerals and Mining - 4: abstracts of Int. Conf. / VNIIOkeangeologia. St. Petersburg, 2014, pp.21-24.

[15] Arykantsev, V. V. (2015) / Underwater studies of traction properties and patency of the walking device MAK-1 / V. V. Arykantsev, V. V. Chernyshev // And News of the southern Federal University. Technical Sciences, - 2015. - № 10. pp. 169-178.

[16] Chernyshev V.V. (2016) / Design and underwater tests of subsea walking hexapod MAK-1 / V.V. Chernyshev, V.V. Arykantsev, A.E. Gavrilov et al. // Proc. of the ASME 2016 35th Int.Conf. on Ocean, Offshore and Arctic Engineering OMAE 2016, Busan, 2016. - 9 p.

[17] Chernyshev V.V. (2017) / Cyclical mechanism of walking with a transformable trajectory of the reference point / V.V. Chernyshev, V.V. Arykantsev // Theory of mechanisms and machines. - 2017. - Vol.15, № 2. - pp. 71-79.

[18] Pat. 2207583 RF B 62 D 57/032. Walking support for cross-country vehicles / Chernyshev V.V., Briskin E.S., Savin A.Yu.; VSTU. (2003).

[19] Davydov O. I., Platonov A. K. (2017) / Robot and Artificial Intelligence. Technocratic approach. //Preprint of the Keldysh IPM RAS, No. 112, 2017, 24 p.

[20] Huang W. H., Beevers K. R. (2004) / Topological Mapping with Sensing-limited Robots. //In Proc. of the 6th International Workshop on the Algorithmic Foundations of Robotics (WAFR), 2004, pp. 367-382.

[21] Nuchter A., Hertzberg J. (2008) / Towards Semantic Maps for Mobile Robots. //Robotics and Autonomous Systems, vol. 56, no. 11, Nov. 2008, pp. 915-926

[22] Davydov O. I., Pryanichnikov V. E. (2015) / Architecture of the mobile service robot control system. //Information-measuring and control systems, №7, vol. 13, 2015, p. 41-50.

[23] Gulati S., Kuipers B. (2008) / High performance control for graceful motion of an intelligent wheelchair. // In Proc. of IEEE International Conference on Robotics and Automation (ICRA) , 2008, pp. 3932-3938.

[24] Davydov O. I., Platonov A. K. (2015) / Algorithm for controlling the differential drive of the mobile robot RB-2. //Preprints of the Keldysh IPM RAS, №25, 2015, 17 p.

[25] Davydov O. I., Platonov A. K. (2015) / complex map of the robot's operating environment. //Robotics and artificial intelligence. Materials of the VII all-Russian scientific and technical conference, Zheleznogorsk, 2015, pp. 108-114.

[26] Davydov O. I., Platonov A. K. (2016) / Database for the operating environment model of a service robot. //Preprints of the Keldysh IPM RAS, №132, 2016, 20 p. .

[27] Davydov O. I., Platonov A. K. (2017) / Database for the semantic model of the operating environment of a mobile service robot. //Preprints of the Keldysh IPM RAS, №7, 2017, 24 p.

[28] Davydov O. I., Platonov A. K. (2015) / Passframe network - a combined model of the mobile robot operating environment. //Preprints of the Keldysh IPM RAS, №15, 2015, 28 p. 
[29] Russell S., Norvig P. (2006) /Artificial intelligence: A modern approach, Moscow: Williams publishing house, 2006, pp. 541-545

[30] Kunze L., Tenorth M., Beetz M. (2010) / Putting People's Common Sense into Knowledge Bases of Household Robots. // In Proc. of the Annual German Conference on Artificial Intelligence, 2010, pp. 151-159.

[31] Lifschitz V. (1987) / On the Semantics of STRIPS. Reasoning about Actions and Plans. // Morgan Kaufmann Publishers Inc., 1987, pp. 1-9.

[32] Blum A., Furst M. (1997) / Fast planning through planning graph analysis. // Artificial intelligence. Vol. 90, 1997, pp. 281-300.

[33] Davydov O. I., Platonov A. K. (2015) / Organization and structure of a complex map of the operating environment of a service robot. //Preprints of the Keldysh IPM RAS, №72, 2015, 28 p.

[34] Kunze L., Tenorth M., Beetz M. (2010) / Putting People's Common Sense into Knowledge Bases of Household Robots. // In Proc. of the Annual German Conference on Artificial Intelligence, 2010, pp. 151-159.

[35] Platonov A. K., Kirilchenko A. A., Pryanichnikov V. E. Stepanov Yu. I., Trubitsin O. N. (1996) / Electronic support of mobile robot control systems // Preprints of the Keldysh IPM RAS, №57, 1996, 32 p.

[36] Noskov V. P., Noskov A.V. (2005) / Navigation of mobile robots by rangefinder images. // Mechatronics, automation, controlling, 2005, №12. M.: publishing house "New technologies", pp. 16-21.

[37] Evseev A. A., Noskov V. P., Platonov A. K. (2008) / Formation of an electronic map for Autonomous movement in an industrial environment. // Mechatronics, automation, controlling, 2008, №2. Moscow: publishing house "New technologies", pp. 41-45.

[38] Nguyen V., Gächter S., Martinelli A., Tomatis N., Siegwart R. A (2007) / Comparison of line extraction algorithms using 2D laser rangefinder for indoor mobile robotics. // Autonomous Robot, Vol. 23, 2007, pp. 97 -111.

[39] Pryanichnikov V. E., Shipovalov E. A. (2019) / Application of graphic accelerators for automatic planning with heuristic search in the state space. // Intelligent adaptive robots (special issue of the journal - Informationmeasuring and control systems, vol. 16, no. 12), Moscow: radio engineering, vol. 14, № 1-2, 2019, ISSN 20700814, pp. 61-67, DOI 10.18127/j20700814-201812-07

[40] Pryanichnikov V. E., Aryskin A. A., Ksenzenko A. Ya., Petrakov M. S., Ignatiev V. A., Helemendik R. V. (2018) / Construction of a service Autonomous mobile robot based on control using logical analysis of the feasibility of operations // Scientific service on the Internet: proceedings of the XX all - Russian scientific conference (1722.09.2018, Novorossiysk), Moscow: Keldysh IPM RAS, 2018. DOI:10.20948/abrau-2018. 